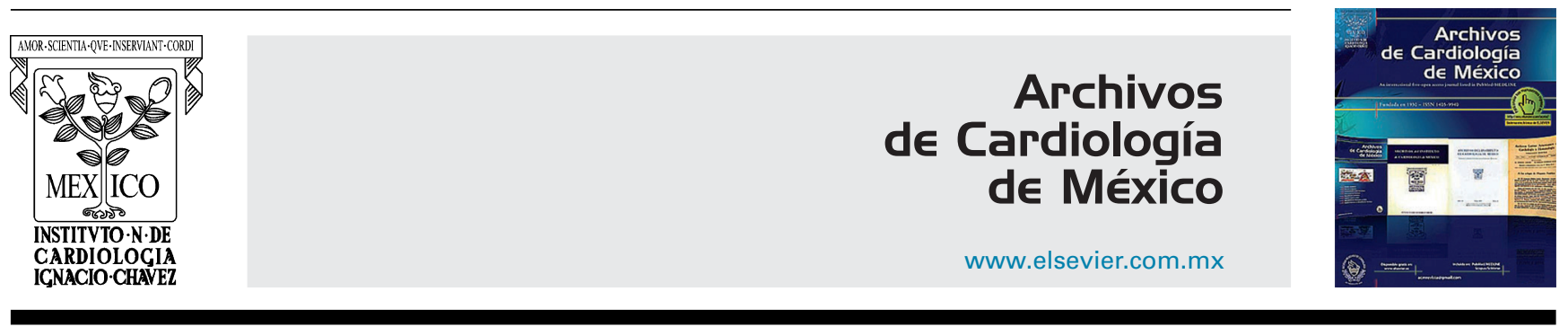

INVESTIGACIÓN CLÍNICA

\title{
Presión arterial en población indígena tzotzil de Chiapas, México
}

\section{Enrique Villarreal-García* y Lourdes Verónica Villarreal-García}

Facultad de Medicina, Universidad Autónoma de Nuevo León, Monterrey, Nuevo León, México

Recibido el 27 de abril de 2017; aceptado el 4 de octubre de 2017

\author{
PALABRAS CLAVE \\ Presión arterial; \\ Diastólica; \\ Sistólica; \\ Indígenas; \\ México
}

\begin{abstract}
Resumen
Objetivo: Determinar las cifras de presión arterial en población indígena tzotzil del estado de Chiapas, México.

Metodología: Estudio transversal descriptivo en población tzotzil de Chenalho del Estado de Chiapas, México, de agosto a diciembre del 2015 en el grupo de 20 a 60 años de edad. Fueron excluidos los pacientes con diagnóstico de diabetes mellitus e hipertensión, y las embarazadas. El tamaño de muestra fue de 205 utilizando como marco muestral el listado de pacientes que acudieron a la cita de control sano en el centro de salud. Se midió la presión arterial sistólica y diastólica por una sola persona; la medición se realizó en el brazo derecho con el paciente en posición sedente, 5 min después de haber ingresado al consultorio, sin haber realizado actividad física previa; se utilizó esfigmógrafo de columna de mercurio marca Adex. El análisis estadístico incluyó promedios, porcentajes e intervalos de confianza para promedios y para porcentajes. Resultados: La presión arterial diastólica promedio es $68.73 \mathrm{mmHg}$ (IC del 95\%, 67.63-69.69), la sistólica $103.65 \mathrm{mmHg}$ (IC del 95\%, 102.24-105.06) y la presión arterial media $80.37 \mathrm{mmHg}$ (IC del 95\%, 79.35-81.39).

Conclusión: Las cifras de presión arterial en población indígena tzotzil de Chiapas, México, son inferiores a las reportadas en la literatura científica.

(c) 2017 Instituto Nacional de Cardiología Ignacio Chávez. Publicado por Masson Doyma México S.A. Este es un artículo Open Access bajo la licencia CC BY-NC-ND (http://creativecommons. org/licenses/by-nc-nd/4.0/).
\end{abstract}

* Autor para correspondencia. Tacaná 12, Colonia Cumbres del Cimatario, Huimilpan, Querétaro de Arteaga, México. Teléfono: 967126 2236.

Correo electrónico: kikevg90_05@hotmail.com (E. Villarreal-García). 


\section{KEYWORDS}

Blood pressure;

Diastolic;

Systolic;

Native;

Mexico

\section{Blood pressure in a native Tzotzil population from the state of Chiapas, Mexico}

\begin{abstract}
Objective: To determine the blood pressure in a native Tzotzil population from the state of Chiapas, Mexico.

Methodology: Cross-sectional study on a Chenalho Tzotzil population from Chiapas, Mexico, from August to December 2015, aged between 20 to 60 years-old. Patients with diabetes mellitus and hypertension were excluded, as well as pregnant patients. The sample size was 205, using as sample basis, the list of patients that went to the healthy patient wellness clinic. The systolic and diastolic pressures were measured by the same person. The measurement was performed on the right arm with the patient seated, five minutes after coming into the clinic, without performing any physical activity beforehand. It was measured with an Adex ${ }^{\top M}$ mercury column sphygmomanometer. The statistical analysis includes mean and percentages, as well as their confidence intervals.

Results: The mean diastolic blood pressure found was $68.73 \mathrm{mmHg}(95 \% \mathrm{Cl}$,-67.63-69.69), with a diastolic of $103.65 \mathrm{mmHg}(95 \% \mathrm{Cl} ; 102.24-105.06)$ and a mean arterial pressure $80.37 \mathrm{mmHg}$ (95\% Cl, 79.35-81.39).

Conclusion: The arterial blood pressure in a Tzotzil native population of Chiapas, Mexico is lower than that reported in the scientific literature.

(C) 2017 Instituto Nacional de Cardiología Ignacio Chávez. Published by Masson Doyma México S.A. This is an open access article under the CC BY-NC-ND license (http://creativecommons. org/licenses/by-nc-nd/4.0/).
\end{abstract}

\section{Introducción}

La presión arterial se refiere a la fuerza de contracción de la sangre generada por el corazón sobre la pared de las arterias para una adecuada perfusión sanguínea sobre el organismo ${ }^{1}$. Se han realizado estudios para determinar el promedio de la presión arterial en la población; dichos estudios plantean el comportamiento natural del organismo. A partir de dichos estudios se establecen parámetros de referencia que tienen implicaciones clínicas en el diagnóstico de ciertas patologías. En este contexto se conocen las cifras de presión arterial establecidas como adecuadas por las asociaciones internacionales ${ }^{1-3}$.

La American Heart Association establece que las cifras de presión arterial se consideran adecuadas cuando los valores sistólicos son menores a $135 \mathrm{mmHg}$ y los diastólicos menores a $85 \mathrm{mmHg}$, aunado a ello propone como valores óptimos $130 / 80^{2}$

De igual forma, la Asociación Europea de Hipertensión establece como parámetros adecuados entre $120 \mathrm{mmHg}$ y $129 \mathrm{mmHg}$ para la sistólica y $80 \mathrm{mmHg}$ y $90 \mathrm{mmHg}$ para la diastólica; también propone valores óptimos y los indica menores a $120 \mathrm{mmHg}$ y a $80 \mathrm{mmHg}^{3}$, respectivamente.

El Séptimo Informe del Joint National Committee on Prevention, Detection, Evaluation and Treatment of High Blood Pressure propone como cifras adecuadas valores inferiores a $120 \mathrm{mmHg}$ para la sistólica y $80 \mathrm{mmHg}$ para la diastólica. En este caso, a diferencia de los señalados inicialmente, no indica valores óptimos ${ }^{4}$.

La guía de práctica clínica de la hipertensión arterial del Instituto Mexicano del Seguro Social propone como valores adecuados aquellos inferiores a $140 \mathrm{mmHg}$ para la presión sistólica y menores a $90 \mathrm{mmHg}$ para la presión diastólica ${ }^{1}$.

Sin embargo, es una realidad que existen grupos específicos de población que no encajan dentro de los parámetros establecidos como adecuados, panorama indicado en las guías diagnósticas terapéuticas. En este contexto, el objetivo del artículo es determinar las cifras de presión arterial en población indígena tzotzil del estado de Chiapas, México.

\section{Metodología}

Se realizó un estudio transversal descriptivo en población tzotzil de Chenalho del Estado de Chiapas, México, en el periodo de agosto a diciembre del 2015. El total de la población de la localidad es 2,562 personas, de ellos 1,261 (50.0\%) pertenecen al grupo etario de 20 a 60 años; del total de la población, 1,337 son mujeres (52.2\%) y 1,225 son hombres (47.8\%).

Se incluyó a hombres y mujeres entre 20 y 60 años de edad que asistieron a la consulta de la unidad médica y que estuvieron de acuerdo en someterse al procedimiento de medición de la presión arterial. Fueron excluidos los pacientes con diagnóstico de diabetes mellitus, hipertensión arterial o embarazo.

El tamaño de muestra $(n=205)$ corresponde al $16 \%$ de la población del grupo etario estudiado (20 a 60 años); se calculó con la fórmula de promedios para población infinita, con un nivel de confianza del $95 \%$ para una zona de rechazo de la hipótesis nula ( $\mathrm{Z}$ alfa $=1.64$ ), asumiendo como hipótesis que el promedio de presión arterial diastólica es $70 \mathrm{mmHg}$. De la muestra, el $45.9 \%$ (94) correspondió al grupo etario de 20 a 30 años, el $24.4 \%$ (50) al grupo de 31 a 40 años, el $14.6 \%$ (30) al grupo de 41 a 50 años y el $15.1 \%$ (31) al grupo de 51 a 60 años. Para la recolección de la muestra se eligió a pacientes que acudieron a la cita de control sano en la unidad médica y que cumplieron con los criterios de selección; cuando el paciente ya había sido incluido en la muestra en las visitas previas no se consideró nuevamente. 
Se estudiaron la edad, el sexo, el peso, la talla, el índice de masa corporal y el estado nutricional determinado a partir del índice de masa corporal (bajo peso, menor a 18.5; normal, de 18.5 a 24.99; sobrepeso, de 25 a 29.99; obesidad, 30 y más).

Se midieron la presión arterial sistólica y la diastólica por una sola persona; la medición se realizó en el brazo derecho con el paciente en posición sedente, 5 min después de haber ingresado al consultorio, sin haber realizado actividad física previa; se utilizó esfigmógrafo de columna de mercurio marca productos Adex S.A. de C.v. ${ }^{1,3,5,6}$. En un momento posterior se determinó la presión arterial media.

El análisis estadístico incluyó promedios, desviación estándar, porcentajes e intervalos de confianza para promedios y para porcentajes.

\section{Resultados}

El 69.3\% (IC del 95\%, 63.0-75.6) de la población estudiada pertenece al sexo femenino y el 30.7\% (IC del 95\%, 24.437.0) al sexo masculino; el promedio u desviación estándar de edad es 36.16 \pm 12.04 años (IC del 95\%, 34.51-37.81), la talla promedio $1.49 \pm 0.08 \mathrm{~m}$ (IC 95\%; 1.48-1.50), el peso $56.55 \pm 10.39 \mathrm{~kg}$ (IC del 95\%, 55.12-57.98) y el índice de masa corporal $25.23 \pm 4.30$ (IC del 95\%, 24.62-25.82).

El 51.2\% (IC del 95\%, 44.4-58.1) de la población se ubica en el estrato nutricional normal y el 35.6\% (IC del 95\%, 29.142.2) en el estrato sobrepeso (tabla 1).

La presión arterial diastólica promedio en la población estudiada es $68.73 \pm 7.94 \mathrm{mmHg}$ (IC del 95\%; 67.63-69.82) (tabla 2).

La presión arterial sistólica, diastólica y media es superior en los hombres que en las mujeres (tabla 3).

En la población con estado nutricional normal, el promedio de presión arterial sistólica es $102.86 \pm 10.35 \mathrm{mmHg}$ (IC del 95\%, 100.85-104.86), la presión diastólica se reporta en $68.76 \pm 8.04 \mathrm{mmHg}$ (IC del 95\%, 67.20-70.32) y la presión

Tabla 1 Prevalencia de estado nutricional en la población estudiada

\begin{tabular}{lllll}
\hline Estado nutricional & Porcentaje & & \multicolumn{2}{c}{ IC del 95\% } \\
\cline { 1 - 1 } \cline { 5 - 5 } \cline { 5 - 5 } & & & Inferior & Superior \\
\hline Bajo peso & 1.5 & & 0.0 & 3.1 \\
Normal & 51.2 & & 44.4 & 58.1 \\
Sobrepeso & 35.6 & & 29.1 & 42.2 \\
Obesidad & 11.7 & & 7.3 & 16.1 \\
\hline
\end{tabular}

El total de la muestra es 205 pacientes.

Tabla 2 Presión arterial sistólica, diastólica y media

\begin{tabular}{llllll}
\hline $\begin{array}{l}\text { Presión } \\
\text { arterial }\end{array}$ & $\begin{array}{l}\text { Promedio } \\
(\mathrm{mmHg})\end{array}$ & $\begin{array}{l}\text { Desviación } \\
\text { estándar }\end{array}$ & & \multicolumn{2}{c}{ IC del 95\% } \\
\cline { 1 - 3 } \cline { 6 - 7 } \cline { 5 - 6 } & & & & Inferior & Superior \\
\hline Sistólica & 103.65 & 10.23 & & 102.24 & 105.06 \\
Diastólica & 68.73 & 7.94 & & 67.63 & 69.82 \\
Media & 80.37 & 7.40 & & 79.35 & 81.39 \\
\hline
\end{tabular}

El total de la muestra es 205 pacientes. arterial media en $80.13 \pm 7.39 \mathrm{mmHg}$ (IC del 95\%, 78.6981.55) (tabla 4).

Los valores de presión arterial diastólica 70.20土 $7.69 \mathrm{mmHg}$ (IC del 95\%, 68.01-72.37) y la presión arterial media $81.73 \pm 7.29 \mathrm{mmHg}$ (IC del 95\%, 79.66-83.81) fueron más altos en el grupo de 31 a 40 años. Para la presión arterial sistólica $105.36 \pm 9.61 \mathrm{mmHg}$ (IC del 95\%, 101.63-109.09), los valores más elevados se presentan en el grupo de 41 a 50 años (tabla 5).

\section{Discusión}

Si bien es cierto se ha establecido por consenso el parámetro de presión arterial promedio de la población a nivel mundial, dicho parámetro no puede enmarcar la totalidad de la población debido a que se excluyen características morfológicas de poblaciones únicas ${ }^{7}$; al respecto, en la literatura ya se ha señalado el papel de la genética ${ }^{8-10}$. La importancia de identificar la presión arterial para grupos específicos estriba en las consecuencias de conocer los parámetros de referencia, valores que en el largo plazo pueden tener consecuencias clínicas para establecer diagnósticos oportunos; de esta manera, se entendería a poblaciones que desarrollan eventos patológicos dentro de los parámetros establecidos como adecuados por el consenso; en ello estriba la importancia del artículo que aquí se presenta, el cual estudia a población indígena tzotzil de Chiapas, México.

Es verdad que como criterio de exclusión se consideró la presencia de hipertensión arterial, embarazo o diabetes, lo cual implica eliminar los valores ubicados en el extremo derecho de la curva; esto no es casualidad, intencionadamente así se diseñó, pero esto no significa que se hayan eliminado los valores extremos en una población que no cursa con las patología o eventos clínicos señalados; en principio, porque el objetivo del artículo es identificar las cifras de presión arterial en la población indígena estudiada, al establecer estos criterios, de facto queda en claro que los resultados solamente son comparables con población que no presente los criterios señalados. Es verdad que se podrían haber incluido pero ese no era el propósito del trabajo.

Considerar como criterio de exclusión a la población mayor de 60 años tiene como fundamento lo propuesto por las guías estadounidenses ${ }^{11}$ y europeas ${ }^{3}$ de hipertensión, las cuales señalan que el comportamiento de la presión arterial después de los 60 años se modifica. De alguna forma, esto es una fortaleza del estudio. También como fortaleza del estudio se puede considerar la toma de presión arterial realizada por una sola persona, lo cual se traduce en la estandarización del método y permite suponer que los valores obtenidos tienen solidez.

La comparación de los valores establecidos como adecuados por los organismos internacionales para la presión sistólica y diastólica se encuentra fuera del IC identificado en la población estudiada, información con la cual se puede afirmar que estadísticamente los valores de la población estudiada son menores que los referidos en la literatura como adecuados ${ }^{3,4,11}$. Aún más, la comparación con las cifras de presión arterial encontradas en este trabajo son inferiores a las reportadas en la Encuesta Nacional de Salud 
Tabla 3 Presión arterial sistólica, diastólica y media en hombres y mujeres

\begin{tabular}{|c|c|c|c|c|c|c|c|c|}
\hline \multirow[t]{3}{*}{ Presión arterial } & \multicolumn{4}{|c|}{ Hombres } & \multicolumn{4}{|c|}{ Mujeres } \\
\hline & \multirow[t]{2}{*}{ Promedio } & \multirow{2}{*}{$\begin{array}{l}\text { Desviación } \\
\text { estándar }\end{array}$} & \multicolumn{2}{|c|}{ IC del 95\% } & \multirow[t]{2}{*}{ Promedio } & \multirow{2}{*}{$\begin{array}{l}\text { Desviación } \\
\text { estándar }\end{array}$} & \multicolumn{2}{|c|}{ IC del $95 \%$} \\
\hline & & & Inferior & Superior & & & Inferior & Superior \\
\hline Sistólica & 106.66 & 10.00 & 104.14 & 109.18 & 102.36 & 10.08 & 100.65 & 103.99 \\
\hline Diastólica & 70.95 & 7.76 & 68.99 & 72.90 & 67.74 & 7.84 & 66.44 & 69.04 \\
\hline Media & 82.85 & 7.23 & 81.03 & 84.67 & 79.27 & 7.23 & 78.07 & 80.47 \\
\hline
\end{tabular}

El total de la muestra es 205 pacientes.

Tabla 4 Presión arterial sistólica, diastólica y media por estado nutricional

\begin{tabular}{|c|c|c|c|c|}
\hline \multirow{2}{*}{$\begin{array}{l}\text { Presión } \\
\text { Arterial }\end{array}$} & \multirow{2}{*}{$\begin{array}{l}\text { Promedio } \\
(\mathrm{mmHg})\end{array}$} & \multirow{2}{*}{$\begin{array}{l}\text { Desviación } \\
\text { estándar }\end{array}$} & \multicolumn{2}{|c|}{ IC del 95\% } \\
\hline & & & Inferior & Superior \\
\hline \multicolumn{5}{|l|}{ Sistólica } \\
\hline Bajo peso & 96.67 & 5.77 & 82.32 & 111.01 \\
\hline Normal & 102.86 & 10.35 & 100.85 & 104.86 \\
\hline Sobrepeso & 104.25 & 9.7 & 101.98 & 106.51 \\
\hline Obesidad & 106.25 & 11.34 & 101.46 & 111.04 \\
\hline \multicolumn{5}{|l|}{ Diastólica } \\
\hline Bajo peso & 63.33 & 5.77 & 48.99 & 77.68 \\
\hline Normal & 68.76 & 8.04 & 67.20 & 70.32 \\
\hline Sobrepeso & 68.08 & 7.57 & 66.31 & 69.85 \\
\hline Obesidad & 71.25 & 8.50 & 67.66 & 74.84 \\
\hline \multicolumn{5}{|l|}{ Media } \\
\hline Bajo peso & 74.44 & 5.09 & 61.80 & 87.09 \\
\hline Normal & 80.13 & 7.39 & 78.70 & 81.56 \\
\hline Sobrepeso & 80.14 & 7.03 & 78.50 & 81.78 \\
\hline Obesidad & 82.92 & 8.35 & 79.39 & 86.45 \\
\hline
\end{tabular}

El total de la muestra es 205 pacientes.

para población mexicana por género, por estado nutricional, grupo etario o área rural ${ }^{12}$.

La implicación clínica de lo señalado se refleja en el diagnóstico de la hipertensión arterial ${ }^{1}$ y de afecciones como la preeclampsia ${ }^{1,13,14}$, las cuales deberían de considerar cifras de presión arterial menores a las indicadas por las guías diagnósticas terapéuticas; no obstante, para esta observación se requiere de otro tipo de estudio que rebasa los límites del artículo en cuestión.

La comparación de las cifras de presión arterial sistólica, diastólica y media en hombres y mujeres señalan valores superiores en el sexo masculino; sin embargo, al respecto se podría cuestionar el tamaño de muestra; no obstante, la demostración estadística de la diferencia a través de los IC descarta la posibilidad de que el tamaño de muestra esté determinando la diferencia. Si se incrementara el tamaño de la muestra, la diferencia se haría más evidente, por lo cual se puede considerar que el comportamiento de la presión arterial entre hombres y mujeres en esta población es diferente. En torno al tema en la literatura, se reportan cifras de presión arterial superiores en hombres en relación con las mujeres ${ }^{12}$, y aunado a ello identifican la menor
Tabla 5 Presión arterial sistólica, diastólica y media por grupo de edad

\begin{tabular}{|c|c|c|c|c|}
\hline \multirow{2}{*}{$\begin{array}{l}\text { Grupo de edad } \\
\quad \text { (años) }\end{array}$} & \multirow{2}{*}{$\begin{array}{l}\text { Promedio } \\
(\mathrm{mmHg})\end{array}$} & \multirow{2}{*}{$\begin{array}{l}\text { Desviación } \\
\text { estándar }\end{array}$} & \multicolumn{2}{|c|}{ IC del 95\% } \\
\hline & & & Inferior & Superior \\
\hline \multicolumn{5}{|l|}{ Sistólica } \\
\hline 20 a 30 & 102.50 & 10.76 & 100.32 & 104.68 \\
\hline 31 a 40 & 104.80 & 9.08 & 102.22 & 107.38 \\
\hline 41 a 50 & 105.36 & 9.61 & 101.63 & 109.09 \\
\hline 51 a 60 & 103.87 & 10.85 & 99.89 & 107.85 \\
\hline \multicolumn{5}{|l|}{ Diastólica } \\
\hline 20 a 30 & 68.33 & 8.62 & 66.69 & 70.18 \\
\hline 31 a 40 & 70.20 & 7.69 & 68.01 & 72.37 \\
\hline 41 a 50 & 67.86 & 7.38 & 64.99 & 70.72 \\
\hline 51 a 60 & 68.06 & 6.54 & 65.66 & 70.46 \\
\hline \multicolumn{5}{|l|}{ Media } \\
\hline 20 a 30 & 79.79 & 8.09 & 78.15 & 81.43 \\
\hline 31 a 40 & 81.73 & 7.29 & 79.66 & 83.81 \\
\hline 41 a 50 & 80.36 & 6.30 & 77.91 & 82.80 \\
\hline 51 a 60 & 80.00 & 6.20 & 77.72 & 82.28 \\
\hline
\end{tabular}

El total de la muestra es 205 pacientes.

prevalencia de la hipertensión en el sexo femenino, pero este es otro tema ${ }^{15}$.

\section{Conclusión}

En conclusión, se puede decir que las cifras de presión arterial en población indígena tzotzil de Chiapas, México, son inferiores a las reportadas como adecuadas en la literatura científica. Por consiguiente, es necesario estandarizar las cifras de presión arterial de poblaciones específicas para vigilar el desarrollo de patologías asociadas al aumento o la disminución de la tensión arterial.

\section{Financiación}

Para la realización de este proyecto de investigación no se contó con financiamiento externo; se utilizaron los recursos de la unidad médica y del personal que lo realizó. 


\section{Conflicto de intereses}

\section{Los autores declaran no tener ningún conflicto de intereses.}

\section{Bibliografía}

1. Consejo de Salubridad Genera. Diagnóstico y tratamiento de la hipertensión arterial en primer nivel de atención. Guía de Práctica Clínica. Centro Nacional de Excelencia Tecnológica en Salud. México 2014 [consultado 14 Ene 2017]. Disponible en: http://www.cenetec.salud.gob.mx/descargas/gpc/ CatalogoMaestro/076-GCP__HipertArterial1NA/HIPERTENSION_ EVR_CENETEC.pdf.

2. Pickering T, Hall J, Appel L, et al. Recommendations for blood pressure measurement in humans and experimental animals: Part 1: Blood pressure measurement in humans: A statement for professionals from the Subcommittee of Professional and Public Education of the American Heart Association Council on High Blood Pressure Research. Hypertension. 2005;45:142-61 [consultado 9 Feb 2017). Disponible en: http://www. peroxfarma.com/files/fck/Pickering.pdf.

3. European Society of Hypertension. European Society of Cardiology guidelines for the management of arterial, hypertension. Guidelines Committee. J Hypertens. 2003;21:1011-53.

4. Chobanian AV, Bakris GL, Black HR, et al., Joint National Committee on Prevention, Detection, Evaluation, and Treatment of High Blood Pressure; National Heart, Lung, and Blood Institute; National High Blood Pressure Education Program Coordinating Committee. Seventh Report of the Joint National Committee on Prevention, Detection, Evaluation, and Treatment of High Blood Pressure: The JNC 7 complete report. Hypertension. 2003;42:1206-52.

5. O'Brien E, Asmar R, Beilin L, et al., on behalf of the European Society of Hypertension Working Group on Blood Pressure Monitoring. European Society of Hypertension recommendations for conventional, ambulatory and home blood pressure measurement. J Hypertens. 2003;21:821-48.
6. O’Brien E, Waeber B, Parati G, et al. Blood pressure measuring devices: Recommendations of the European Society of Hypertension. BMJ. 2001;322:531-6.

7. Bogin B, Rios L. Rapid morphological change in living humans; implications for modern human origins. Comp Biochem Physiol A Moll Integr Physiol. 2003;136:71-84.

8. Rafiq S, Anand S, Roberts R. Genome - wide association studies of hypertension: Have they been fruitful? J CardiovascTransl Res. 2010;3:189-96.

9. O'Shaughnessy KM. The genetics of essential hypertension. Br J Clin Pharmacol. 2001;51:5-11.

10. Ramírez-Bello J, Pérez-Méndez $\mathrm{O}$, Ramírez-Fuentes $\mathrm{S}$, et al. Genética y genómica de la hipertensión arterial: una actualización. Arch Cardiol Méx. 2017;81:240-50 [consultado 14 Feb 2017]. Disponible en: http: //www.scielo.org. $\mathrm{mx} /$ scielo.php?script=sci_arttext\&pid= S1405-99402011000300013\&lng=es.

11. Pickering TG, Hall JE, Appel LJ, et al. Recommendations for blood pressure measurement in humans and experimental animals: Part 1: Blood pressure measurement in humans: A statement for professionals from the Subcommittee of Professional and Public Education of the American Heart Association Council on High Blood Pressure Research. Hypertension. 2005;45:142-61.

12. Barquera S, Campos-Nonato I, Hernández-Barrera L, et al. Hypertension in Mexican adults: Results from the National Health and Nutrition Survey 2006. Salud Pública Méx. 2010;52 Suppl 1:S63-71 [consultado 12 Feb 2017]. Disponible en: http: / / www.scielo.org. mx/scielo.php?script=sci_arttext\&pid= S0036-36342010000700010\&lng=es.

13. González-Navarro P, Martínez-Salazar GG, García-Nájera O, et al. Preeclampsia, eclampsia y HELLP. Rev Mex Anest. 2015;38:S118-27.

14. Robrts JM, August PA, Bakris G, et al. Hypertension in pregnancy. Report of the American College of Obstetricians and Gynecologists' Task Force on Hypertension in Pregnancy. Obstet Gynecol. 2013;122:1122-31 [Dataset].

15. Instituto Nacional de Salud Pública. Encuesta Nacional de Salud 2000. México: Secretaría de Salud; 2000. 